\title{
A theoretical model of emotion processing for optimizing the cost function of discrepancy errors between wants and gets
}

\section{David Tam}

Address: Department of Biological Sciences, University of North Texas, Denton, TX 76203, USA

Email: David Tam - dtam@unt.edu

from Eighteenth Annual Computational Neuroscience Meeting: CNS*2009

Berlin, Germany. 18-23 July 2009

Published: I 3 July 2009

BMC Neuroscience 2009, I0(SuppI I):PII doi:I0.II86/I47I-2202-I0-SI-PI I

This abstract is available from: http://www.biomedcentral.com/I47I-2202/I0/SI/PI I

(c) 2009 Tam; licensee BioMed Central Ltd.

A number of theoretical model of emotional processing were derived previously $[1,2]$ called EMOTION-I and EMOTION-II from the basic evolutionary principles of survival function in a real-world model of the interaction between an autonomous agent (organism or autonomous robot) and its environment. An associative reinforcementlearning model was used to establish the innate and acquired behaviors for evaluating the survivability of the agent with respect to the environment. Discrepancies (errors) always exist between the intended actions and the actual actions, and between the perceived sensory inputs and actual inputs in a real-world environment. Emotions emerge as the emergent property that assesses the accuracy of this internal model for predicting its interactions with the environment. Happy emotion results from an accurate model with a congruent match between the subjective reality and objective reality, and conversely for unhappy emotions.

This paper extends the previous models to describe the process for the optimization process to derive a solution to minimize the discrepancy error between the predicted desirable outcome and the actual outcome in the real world. The time-derivative of the error signal provides a mechanism for assessing how fast the cost function approaches, whereas the second time-derivative (rate of change) provides the direction in which the minimization vs. maximization of the cost function may occur. These assessment measures serve as the emotional context in which an organism can use to guide its action to correct either its internal model (self) or change the world as it see fits (subjective reality) if what it perceived did not fit its internal model of prediction. Various sub-emotions are described in this theoretical emotional model to account for these computational phenomena.

\section{References}

I. Tam D: EMOTION-I Model: A biologically-based theoretical framework for deriving emotional context of sensation in autonomous control Systems. The Open Cybernetics \& Systemics Journal 2007, I:28-46.

2. Tam D: EMOTION-II Model: A theoretical framework for happy emotion as a self-assessment measure indicating the degree-of-fit (congruency) between the expectancy in subjective and objective realities in autonomous control systems. The Open Cybernetics \& Systemics Journal 2007, 1:47-60. 